\title{
Etude de la dynamique spatio-temporelle de la forêt des Ait Daoud ou Ali (Haut Atlas central, Maroc) en utilisant les techniques géospatiales
}

\author{
Study of the spatio-temporal dynamics of the Ait Daoud ou Ali (Central High \\ Atlas, Morocco) Forest using geospatial techniques
}

\section{Estudio de la dinámica espacio-temporal del bosque de Ait Daoud ou Ali (Alto Atlas Central, Marruecos) mediante técnicas geoespaciales}

\author{
Aïman Hili \\ a.hili@usms.ma 0000-0003-2220-6360 \\ Rachid Bissour \\ bissour1983@usms.ma 0000-0002-5391-9921 \\ Farid Jaa \\ farid.jaa@usms.ac.ma@0000-0002-7158-5080 \\ Hanane Reddad \\ h.reddad@usms.ma 0000-0002-6238-165X \\ Yassine El Jouhary \\ yassineeljaouhary02@gmail.com @ 0000-0002-1534-4944
}

Faculté des Lettres et des Sciences Humaines, Université Sultan Moulay Slimane.

Avenu Ibn khaldoun, B.P. 524 Beni Mellal, Maroc

INFO ARTÍCULO

A reçu: 04-07-2021

Revu: 21-01-2022

Accepté: 21-01-2022

\section{MOTS CLÉS}

Classification supervisée

NDVI

Étude diachronique La forêt des Ait Daoud ou Ali Haut Atlas central

Maroc

\section{RESUME}

Depuis longtemps l'Homme cherche l'exploitation des ressources naturelles à des fines quotidiennes. Ainsi, le développement scientifique et technologique actuel lui a facilité et lui a donné des vraies opportunités pour la surexploitation de ces ressources en général et du couvert végétal en particulier. Afin de montre cette exploitation nous voulons, dans le présent article, étudier l'évolution du couvert végétal de la forêt des Ait Daoud ou Ali, située dans le Haut Atlas central sur une superficie de 30860 ha, entre 1972, 1999 et 2020, en se basant sur la télédétection et l'outil SIG. Pour ce qui concerne la méthodologie de travail, l'étude multi-chronique de la dynamique du couvert végétal de la zone d'étude, repose sur l'analyse spatio-temporelle et le traitement des images satellitaires par la télédétection spatiale. En effet, dans le présent papier nous avons opté cette démarche qui s'appuie sur l'utilisation des données des images du satellite de LandSat de trois types; il s'agit des images de type MSS, ETM et Oli, pour trois dates 1972, 1999 et 2020 (période de 48 ans) pour identifier les différences spatiales du couvert végétal entre les trois dates choisies. En effet, l'étude de la dynamique du couvert végétal de la forêt des Ait Daoud ou Ali entre 1972, 1999 et 2020, a montré une variation spatio-temporelle très importante, liée essentiellement à plusieurs facteurs (naturels et humaines). La comparaison des résultats de la classification des images Landsat, a montré un recul et une dégradation importante du couvert végétal entre 1972 et 1999 de $12 \%$ de la surface totale de la zone d'étude. Cette dégradation est justifiée par l'intervention de plusieurs facteurs, notamment les conditions climatiques et les périodes sèches qu'a connu le Maroc à cette période. Pour la deuxième période (1999-2020), le traitement a montré une progression du couvert végétal de l'ordre de $10 \%$. Dans cette étude, nous avons mis en évidence l'impact des facteurs naturels et notamment les actions anthropiques sur la dynamique de la forêt des Ait Daoud ou Ali située dans Haut Atlas Central, en se basant sur l'utilisation des techniques géospatiales qui nous ont permis d'avoir des informations très précises relatives à la dynamique spatio-temporelle du couvert végétal. 


\section{KEYWORDS}

Supervised classification
NDVI

Diachronic study

The Ait Daoud ou Ali Forest

Central High Atlas

Morocco

\section{PALABRAS CLAVE}

Clasificación supervisada

NDVI

Estudio diacrónico

Bosque de Ait Daoud ou Ali

Alto Atlas Central

Marruecos

\section{ABSTRACT}

For a long time, Man has been looking for the exploitation of natural resources for daily purposes. Thus, the current scientific and technological development has facilitated and given him real opportunities for the overexploitation of these resources in general and the plant cover in particular. In order to show this exploitation, we want, in this article, to study the evolution of the vegetation cover of the forest of Ait Daoud or Ali, located in the Central High Atlas on an area of 30860 ha, between 1972, 1999 and 2020, based on remote sensing and GIS tool. Regarding the methodology of work, the multi-chronic study of the dynamics of the vegetation cover of the study area, is based on the spatio-temporal analysis and processing of satellite images by remote sensing space. Indeed, in this paper we have opted for this approach which is based on the use of data from LandSat satellite images of three types; these are the images of type MSS, ETM and Oli, for three dates 1972, 1999 and 2020 (period of 48 years) to identify the spatial differences of the vegetation cover between the three selected dates. Indeed, the study of the dynamics of the vegetation cover of the forest of Ait Daoud or Ali between 1972, 1999 and 2020, showed a very important spatio-temporal variation, linked essentially to several factors (natural and human). The comparison of the results of the classification of Landsat images, showed a decline and a significant degradation of vegetation cover between 1972 and 1999 of $12 \%$ of the total area of the study area. This degradation is justified by the intervention of several factors, including climatic conditions and dry periods that Morocco has experienced in this period. For the second period (1999-2020), the treatment showed an increase in vegetation cover of about $10 \%$. In this study, we have highlighted the impact of natural factors and especially anthropic actions on the dynamics of the forest of Ait Daoud or Ali located in the Central High Atlas, based on the use of geospatial techniques that have allowed us to have very precise information on the spatio-temporal dynamics of plant cover.

\section{RESUMEN}

Durante mucho tiempo, el hombre ha tratado de explotar los recursos naturales para fines cotidianos. Así, el desarrollo científico y tecnológico actual ha facilitado y le ha dado oportunidades reales para la sobreexplotación de estos recursos en general y de la cubierta vegetal en particular. Para mostrar esta explotación queremos, en el presente artículo, estudiar la evolución de la cubierta vegetal del bosque de Ait Daoud o Ali, situado en el Alto Atlas central sobre una superficie de 30.860 ha, entre 1972, 1999 y 2020, basándonos en la teledetección y la herramienta SIG. En cuanto a la metodología de trabajo, el estudio multicrónico de la dinámica de la cubierta vegetal de la zona de estudio se basa en el análisis espacio-temporal y el procesamiento de imágenes de satélite por teledetección espacial. En este trabajo, hemos optado por este enfoque, que se basa en la utilización de datos de imágenes de satélite LandSat de tres tipos: imágenes MSS, ETM y Oli, para tres fechas 1972, 1999 y 2020 (período de 48 años), con el fin de identificar las diferencias espaciales de la cubierta vegetal entre las tres fechas seleccionadas. En efecto, el estudio de la dinámica de la cubierta vegetal del bosque de Ait Daoud o Ali entre 1972, 1999 y 2020, mostró una variación espacio-temporal muy importante, esencialmente ligada a varios factores (naturales y humanos). La comparación de los resultados de la clasificación de las imágenes Landsat mostró una importante disminución y degradación de la cubierta vegetal entre 1972 y 1999 del $12 \%$ de la superficie total del área de estudio. Esta degradación se justifica por la intervención de varios factores, especialmente las condiciones climáticas y los periodos de sequía que vivió Marruecos durante este periodo. En el segundo período (1999-2020), el tratamiento mostró un aumento de la cubierta vegetal de alrededor del 10\%. En este estudio, hemos puesto de relieve el impacto de los factores naturales y, en particular, de las acciones antrópicas en la dinámica del bosque de Ait Daoud o Ali, situado en el Alto Atlas Central, basándonos en el uso de técnicas geoespaciales que nos han permitido disponer de información muy precisa sobre la dinámica espacio-temporal de la cubierta vegetal. 


\section{INTRODUCTION}

Depuis longtemps, l'Homme cherchait à exploiter les ressources naturelles à des fins quotidiens (Bouiadjra et al., 2011; Labhar, 1998) pour assurer sa vie et ses besoins. Ainsi, le développement scientifique et technologique actuel lui a facilité et lui a donné des vraies opportunités pour la surexploitation des ressources naturelles en général et du couvert végétal en particulier. Pour cela, la protection des ressources naturelles en générale et la ressource forestière en particulier est devenue aujourd'hui, dans le contexte global des changements climatiques et l'émergence de la notion de développement durable, un défi majeur à travers la proposition des lois (loi 11-03, loi 12-03 et loi 22-07) et aussi par la création des aires protégées et des parcs nationaux (Hili \& El Khalki, 2017). Cela venait dans le but de créer un certain équilibre bioécologique et de faire face aux changements climatiques que ce soit à l'échelle globale ou locale.

Vu l'apport de la télédétection spatiale et des outils SIG, notamment dans le croisement des cartes, la détection des zones de dégradation et les zones de progression de la forêt, l'utilisation de ces outils est devenue très intéressante dans les études de la dynamique spatio-temporelle du couvert végétal (Hili \& Abdelaziz, 2021; Gansaonré et al., 2020; Bissour et al., 2018; Sbai et al., 2018; Bouzekraoui et al., 2016; Daoui \& Fatemi, 2014; Brou, 2010; Mahamane et al, 2007.; Hountondji et al., 2004; Nguessan, et al., 2003; Demaze, 2003).

En effet, les études qui ont été réalisées sur la dynamique du couvert végétal (Hili \& Abdelaziz, 2021; Gansaonré et al., 2020; Bissour et al., 2018; Sbai et al., 2018; El Mazi et al., 2018; Raogo, 2018; Diop et al., 2018; Bouzekraoui et al., 2016; Daoui \& Fatemi, 2014; Soro et al., 2014; Tra Bi, 2013; Bouiadjra et al., 2011; Dawson et al., 2011; Brou, 2010; Mahamane et al., 2007; Demarteau et al., 2007; Hammi, 2007; Ouloukoi et al.; 2006; Jeffrey et al., 2006; Cabid et al., 2005; Hountondji et al., 2004; Carmen Salazar Conde et al., 2004; Nguessan, et al., 2003; Demaze, 2003; Dupouey et al., 1999; Labhar, 1998; Thimonier, 1994; Benabid, 1985; Lecompte, 1986; Michel \& Ruelan, 1967), ont montré que ce dernier est souvent influencé par plusieurs facteurs, notamment le climat, la topographie (les pentes, l'exposition des versants et les altitudes) la lithologie, la pédologie, et bien entendu les actions anthropiques que ce soit conscientes ou inconscientes.

Pour ce fait, nous proposons cette étude multi-chronique de la forêt des Ait Daoud ou Ali, située dans le Haut Atlas central calcaire (Royaume du Maroc). Vu que cette région d'étude se situe dans le Haut Atlas central et caractérisée par un contexte naturel et humain très diversifié, que ce soit du point de vue géographique, topographique, géologique, climatique et anthropique, ou aussi de point de vue de sa couverture végétale.

L'objectif de ce travail est l'application des techniques de traitement géospatial sur des données géographiques, et l'évaluation de l'apport de la télédétection spatiale pour la quantification de la dynamique spatio-temporelle du couvert végétal de la forêt des Ait Daoud ou Ali sur une période de quarante-huit ans entre 1972, 1999 et 2020. Cette approche méthodologique consiste à détecter les changements (régression ou progression) dans le couvert végétal, à partir du calcul de l'Indice de Végétation par Différence Normalisé, appelé aussi NDVI (Normalized Difference Vegetative Index), et d'une classification supervisée des images satellitaires de différentes dates (1972, 1999 et 2020). Ainsi, les résultats obtenus dans cette étude et d'autres études venions par la suite (sur la commune d'Aghbala (Hili et al., 2021) et sur la forêt des Ait Bouzid), vont nous permettre d'évaluer l'efficacité des lois et programmes réalisés pour la protection et le sauvegarde du couvert végétal forestier marocain en général et de la zone d'étude en particulier.

\section{LA ZONE DE L’ETUDE}

Sur le plan administratif, la forêt des Ait Daoud ou Ali fait partie de la région de Beni Mellal-Khénifra (carte 1), elle s'étend sur six communes rurales, il s'agit des communes de Larbaa n'Ait Ouqabli, commune de Tiffert n'Ait Hamza et Tagleft (couvrant la majeure partie de la zone de l'étude), et partiellement sur les communes de Isekssi, Tabaroucht et la commune de Boutferda. En général, la forêt est délimitée et côtoie dans sa partie Est par la forêt des Ait Abdi, au Sud par les deux forêts d'Anergui et des Ait Isha et à l'Ouest par la forêt des 
Ait Atta Noumallou. Superficiellement, elle couvre sur une superficie de 53785 ha, et classée parmi les forêts les plus importantes de la région (Direction Régional des Eaux et Forêt, Beni Mellal., 2010). En effet, la forêt abrite une population légale de l'ordre de 21220 habitants (Haut-Commissariat au Plan (HCP), 2014). La zone la plus peuplée au niveau du territoire de la forêt est celui situé au centre de la commune de Tagleft, suivi par le centre de la commune de Larbaa n'Ait Ouqabli. Cette situation confère à la forêt des conditions très favorables à la fois à l'évolution et à la dégradation des ressources forestières.
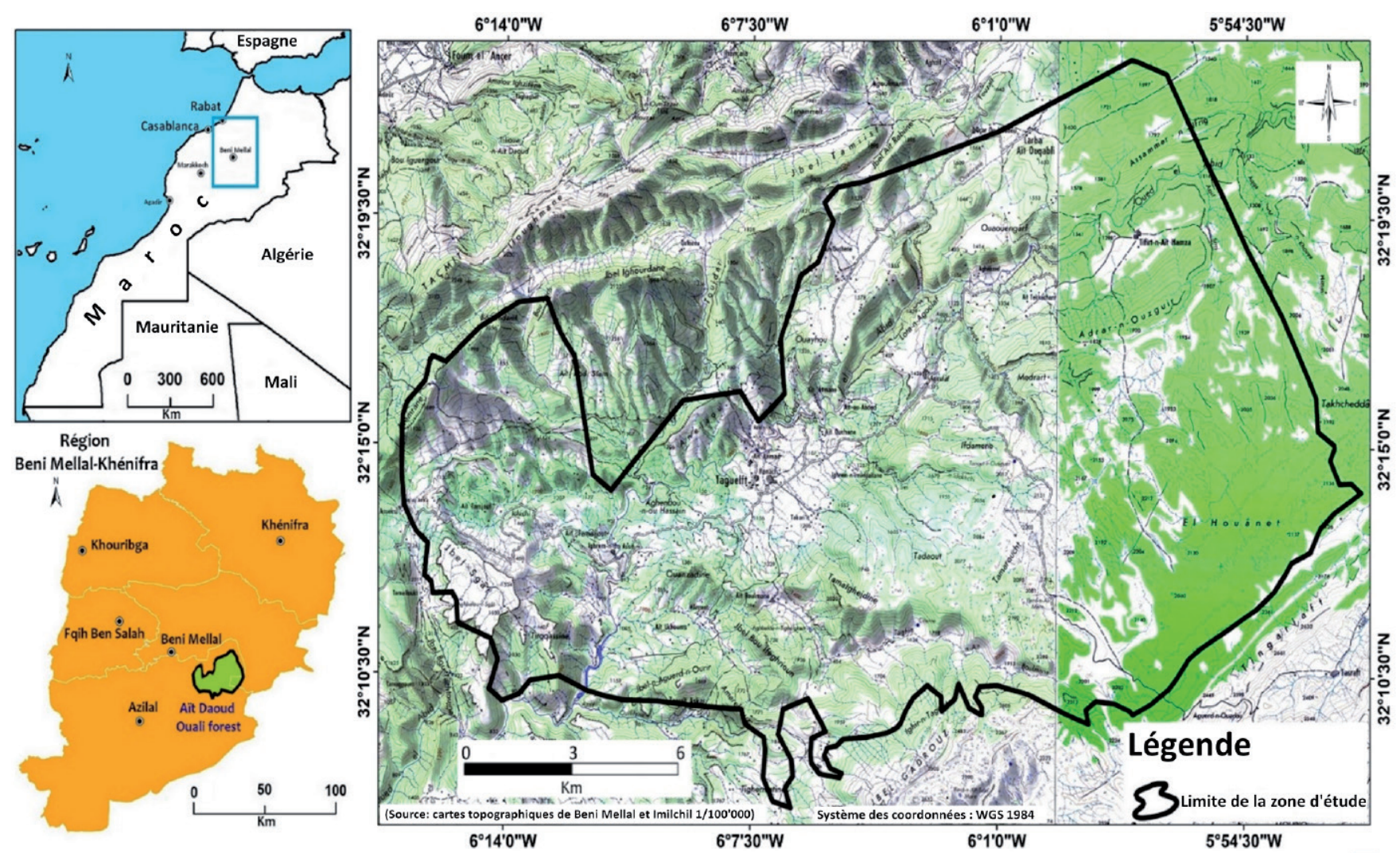

Carte 1. Situation géographique de la forêt des Ait Daoud ou Ali. Source: Élaboration des auteurs

En effet, la spatialisation de la dynamique de la forêt est prouvée par l'influence de plusieurs facteurs naturels et anthropiques. Concernant les conditions topographies, notre terrain d'étude se situe au centre du Haut Atlas Central, sur une tranche altitudinale comprise entre 893 m et 2137 m, qui est marquée souvent par l'accidentée de ces versants. L'analyse de la base de données de la carte des tranches d'altitude montre la dominance des tranches qui varient entre $1500 \mathrm{~m}$ et plus de $2100 \mathrm{~m}$, avec une superficie de l'ordre de 49.6\%. Ces altitudes s'étendent notamment sur la partie Sud-Est de la zone de l'étude. Les reliefs les plus remarquables dans lesquels s'étend la forêt des Ait Daoud ou Ali sont le synclinal de Tagleft, le plateau d'El Houanet et les vallées et les gorges de l'Oued El Abid. La densité du couvert végétal de la forêt est souvent considérable seulement au niveau des versants d'orientation Nord et Nord-Ouest, et au long de la vallée profonde de l'Oued El Abid, et quelques vallées de ses affluents (Aqqa n'Tzanit). Alors que, les terrains presque plats sont souvent occupés par des champs de cultures (Bissour, 2019). Cette diversité de reliefs et de topographie a joué et joue encore un rôle très important et primordial dans la répartition spatiale du couvert végétale et des essences forestières de la forêt des Ait Daoud ou Ali.

Ainsi, l'analyse des classes des pentes qui occupent la zone de la forêt, nous permet de déceler une quasi dominance des pentes moyennes et faibles avec, respectivement, $36 \%$ et $26 \%$ de la superficie totale de la zone de l'étude. Alors que la classe des pentes forte et très fortes n'occupe que $38 \%$ de la surface de la zone. Quant à l'exposition des versants, les versants les plus dominants sont les versants exposés vers le Nord et vers l'Est, le Sud et vers le Sud-Est. Ces versants représentent $52.7 \%$ de la superficie totale de la forêt. 
D'un point de vue géologique, le contexte géologique du terrain d'étude est marqué par la prédominance des formations jurassiques perméables, constituées essentiellement par des sédiments détritiques continentaux (les couches rouges de Bathonien), notamment au niveau du synclinal de Tagleft (Bissour, 2019). Les couches Bajociennes sous-jacentes constituent un aquifère important qui draine les eaux du secteur des Ait Ouqabli. Ce bassin est séparé de celui de Ouaouizerht par le seuil de Jbel Bou Tachtuine-Abbadine, qui est une ride anticlinale complexe et étroite, de direction Nord-Sud (Monbaron, 1982), se déversant vers l'Ouest. Les couches rouges des deux bassins se redressent et se terminent en biseau à l'approche de ce seuil (Bahzad, 1982). Dans la partie Est de la forêt des Ait Daoud ou Ali, le terrain, qui correspond au plateau d'El Houanet, est dominé par des couches calcaires marneux très gélifs et karstiques, ce qui a favorisé et favorise encore le développement d'une multitude de formes karstiques de petites tailles et d'autres de grandes à très grandes tailles (Couvreur, 1988).

Pour ce qui concerne le climat, vu l'effet direct du climat sur l'évolution et la distribution de couvert végétal (Sbai et al., 2018; Dawson et al., 2011; Brou, 2010; Hountondji et al., 2004; Lecompte et Alexandre, 1996), l'analyse climatique semble très importante dans ce genre d'étude, pour cela nous avons utilisé les données de la station des Ait Ouchène (la station des Ait Ouchène est installée dans la zone de la forêt, près du centre de Tagleft sur l'Oued El Abid, mais ne mesure pas les données de la température), et les données de la station de Tillouguit (située au Sud de la forêt, au niveau du centre de la commune de Tilouguit et sur l'Oued Ahançal, l'un des principaux affluents de l'Oued El Abid), pour une période de 30 ans (entre 1986 et 2016). Selon la classification d'Emberger, les deux stations montrent que la zone de la forêt appartient à l'étage bioclimatique de végétation semi-aride avec un hiver frais (pour la station des Ait Ouchène à Tagleft) et hiver très froid pour la station de Tilouguite (Combe, 1977; Couvreur, 1988), alors que la partie Est de la forêt qui s'étend sur le plateau d'El Houanet le climat est de type Sub-Humide avec un hiver très froid (Bissour, 2019). La méthode de Bagnouls et Gaussen (1957) appliquée sur la série de données thermométriques et pluviométriques fournies par la station de Tilouguite, pour mettre en évidence les mois humides et les mois secs, en faisant une analyse fréquentielle mensuelle, montre que les mois secs s'étalent d'Avril à Octobre avec des fréquences dépassant 54\%, et que les années qui présentent 6 et 9 mois secs sont les plus fréquentes (26\%) (Bissour \& El Khalki, 2018).

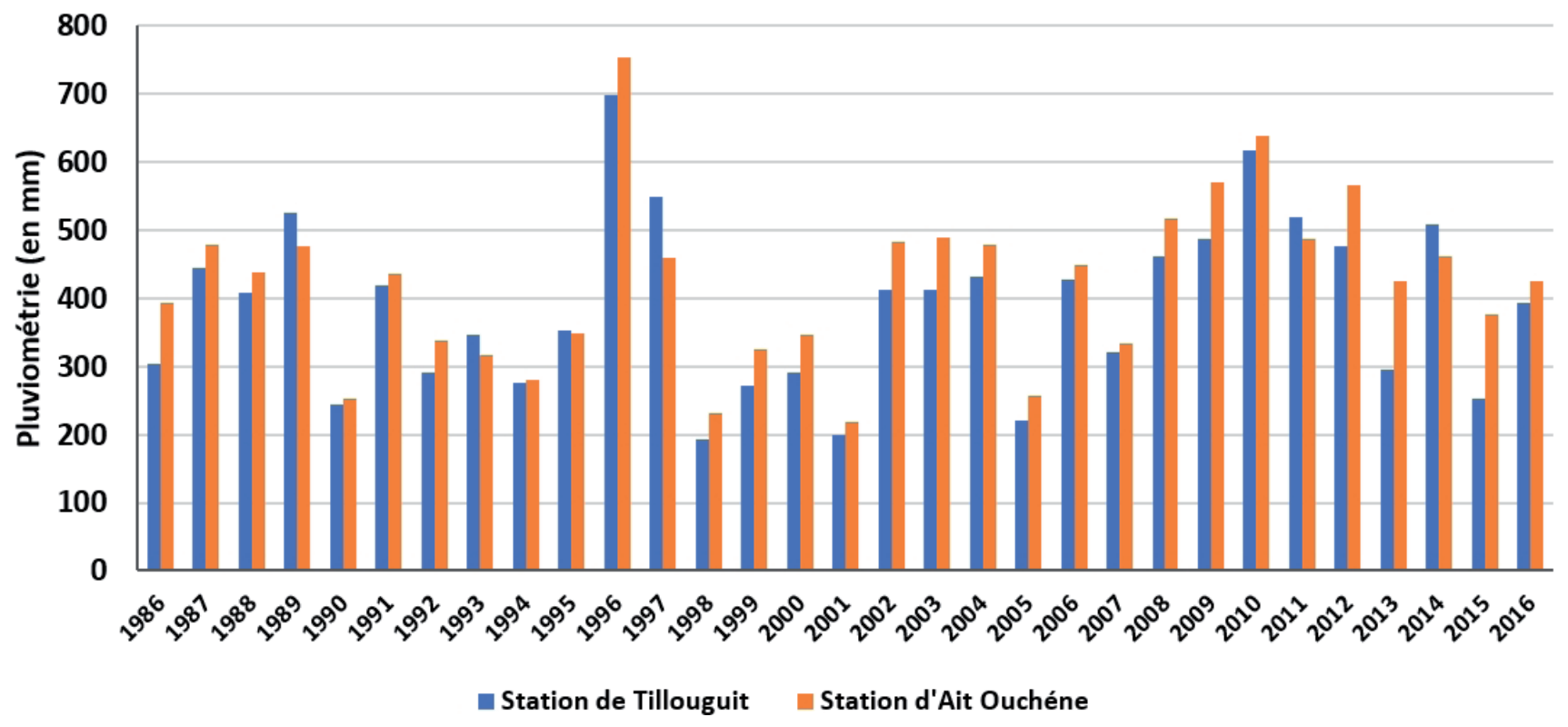

Figure 1. Evolution de la pluviométrie moyenne annuelle aux stations de Tillouguit et Ait Ouchène entre 1986 et 2016. Source: Élaboration des auteurs. 
L'analyse des données de précipitations annuelles des stations étudiées montre une variation importante des précipitations interannuelles (figure 1), où la moyenne interannuelle enregistrée au niveau de la station de Tillouguite atteint $379 \mathrm{~mm}$ et la station des Ait Ouchène enregistre durant la même période $420.7 \mathrm{~mm}$. Ainsi, les valeurs annuelles les plus élevées pour les deux stations sont enregistrées pendant les deux années 1996 et 2010, avec respectivement $697.6 \mathrm{~mm}$ et $616.8 \mathrm{~mm}$ à la station de Tillouguite, et $752.5 \mathrm{~mm}$ et $639.2 \mathrm{~mm}$ à la station des Ait Ouchène. Il faut noter aussi que la zone de la forêt des Ait Daoud ou Ali reçoie annuellement des masses très importantes de la neige qui durent plusieurs mois, notamment au niveau du plateau d'El Houanet qui est dominé par des monts et des crêtes dépassant 2600m d'altitude.

En effet, la relation entre ces facteurs est très intime et directe vu qu'il y a un impact sur l'évolution du couvert végétal. Concernant le climat, elles agissent d'une manière directe sur la répartition du couvert végétal et par conséquent sur leur stabilité. L'impact du choc des gouttes d'eau ouvre la voie au ruissellement par une série de modifications de l'état de surface du sol, au fil de temps l'effet de ces modifications devient de plus en plus importants, avec la pente les effets du ruissellement deviennent plus graves, et menacent par conséquent la stabilité et la fixation des sols et de la végétation.

Tous ces facteurs, i) anthropiques (densité de la population et ses activités) et ii) naturels tel que le climat, la topographie, la lithologie ont contrôlé et contrôlent d'une manière ou autre et interviennent à la distribution spatiale et la dynamique du couvert végétal à l'échelle de la forêt des Ait Daoud ou Ali et les autres zones forestières du Haut Atlas central.

\section{METHODOLOGIE}

\subsection{Données utilisées}

Généralement, l'étude multi-chronique de la dynamique du couvert végétal ou forestier, repose sur l'analyse spatio-temporelle et le traitement des images satellitaires par la télédétection spatiale. En effet, dans le présent papier nous avons opté cette démarche qui s'appuie sur I>utilisation des données des images du satellite de LandSat de trois types; il s'agit des images de type MSS, ETM et Oli, pour trois dates (période de 48 ans) (tableau 1). Ainsi, l'utilisation conjointe de la technique de télédétection spatiale et les outils SIG nous permet i) d'identifier les différences spatiales entre les trois dates choisies (1972, 1999 et 2020) et ii) de valider les résultats de classification obtenus.

En effet, pour toute étude multi-chronique de la végétation, il est très intéressant de bien choisir la date de prise de vue, parce que les caractéristiques du terrain varient souvent d'une année à l'autre et d'une saison à l'autre dans la même année. Donc, la difficulté dans ce genre d'étude c'est de distinguer et d'identifier la végétation naturelle notamment forestière. Pour ce fait, et afin d'augmenter la précision spatiale et radiométrique nous avons i) choisi la période de la saison sèche (de juin à septembre), parce que la majorité des cultures (notamment les céréalicultures) durant cette saison deviennent sèches; ainsi, ii) l'absence des nuages durant cette période sèche (de juin à septembre) rend la qualité des images satellites acquises plus élevée.

Tableau 1. Caractéristiques des images satellites utilisées.

\begin{tabular}{|l|c|c|c|c|}
\hline \multicolumn{1}{|c|}{ Nom du satellite } & Date acquisition & Range & Path & Résolution spatiale \\
\hline MSS (LANDSAT 1) & $08 / 09 / 1972$ & 38 & 216 & 30 \\
\hline ETM (LANDSAT 5) & $27 / 07 / 1999$ & 38 & 201 & 30 \\
\hline OLI (LANDSAT 8) & $08 / 06 / 2020$ & 38 & 201 & 30 \\
\hline
\end{tabular}

Source: Élaboration des auteurs 
Afin de justifier les résultats de l'évolution spatio-temporelle du couvert végétal de la forêt des Ait Daoud ou Ali, nous avons élaboré une carte de degré de risque de la dégradation potentiel (carte 4). L'élaboration de cette carte a été i) appuyée sur la superposition de toutes les cartes des facteurs naturels (tel que la cartes des pentes, des tranches d'altitude, d'exposition des versants (cartes topographiques Beni Mellel et Imilchil $1 / 100^{\prime} 000$ ), carte de la spatialisation des précipitations (données de 4 stations cimatiques pour une période de 31 ans), de la lithologie (carte géologique de Beni Mellal et Imilchil 1/100'000) et la carte du couvert végétal (les images LandSat)), ayant une influence directe et indirecte sur l'évolution du couvert végétal, ainsi cette carte ii) classe la zone de l'étude en zones à risque allant de risque faible au risque très élevé. Dans l'objectif de vérification des résultats, nous avons utilisé cette carte comme référence cartographique pour valider sur le terrain les zones de progression et les zones de recul réelles.

Pour monter l'impact des pratiques humaines sur la dynamique du couvert forestier de la forêt des Ait Daoud ou Ali, nous avons préparé et rempli 120 questionnaires, destiné à la population locale ayant un contact direct avec la forêt et aux gestionnaires de la forêt (Direction Régionale des Eaux et Forêts, la commune, Direction provinciale de l'Agriculture).

\subsection{Les aspects méthodologiques}

Avant de procéder à la classification des trois images satellitaires retenues, et pour assurer un bon seuillage et une bonne visualisation des images, lors des traitements, nous avons fait quelques corrections radiométriques. Par la suite, nous avons élaboré deux types de composition colorées des images satellitaires, la première concerne la composition fausse colorée, qui permet de mettre en évidence le couvert végétal (il apparait en rouge), et la deuxième composition a pour but la visualisation d'une image en couleurs naturelles (RVB = Rouge, Vert, Bleu), afin de l'utiliser dans la classification sous un logiciel de de télédétection et/ou SIG.

Nombreuses sont les méthodes utilisées dans les études de classification de la dynamique du couvert végétal: I'Indice de Végétation par Différence Normalisée (NDVI: Normalised Difference Vegetation Index), Indice de Végétation Résistante à l'Atmosphère (ARVI: Atmospherically Resistant Vegetation Index), Indice de Surveillance de l'Environnement Mondial (GEMI: Global Environment Monitoring Index), Indice de Végétation Ajustée au Sol Transformé (STAVI: Tranformed Soil Adjusted Vegetation Index); Indice de Végétation Ajustée au Sol Modifié (MSAVI: Modified Soil Adjusted Vegetation Index), (Qi et al., 1994; Rondeaux et al., 1996; Bouiadjra et al., 2011). Par contre, dans notre travail, nous avons procédé à calculer l'Indice de Végétation par Différence Normalisée (NDVI) afin de bien identifier les zones d'extension du couvert végétal et forestier pour les différentes dates choisies. Ainsi, la méthode de classification adoptée pour cette étude est celle de la classification supervisée (ou dirigée), et pour éliminer les très petits polygones de classification et améliorer les résultats de classification nous avons procédé à la méthode de (Maximum Likelihood). Quant aux classes choisies pour la classification supervisée, nous avons retenu deux classes: le couvert végétal et le sol nu. Pour valider les résultats de la classification nous avons calculé la séparabilité entre les deux classes pour éliminer toute confusion entre les deux classes. Le niveau de séparabilité obtenu entre les deux classes est très satisfaisant. En outre, pour mener à bien la classification supervisée des images et obtenir des résultats avec haute précision nous avons procédé à i) la vérification des différentes classes sur le terrain (plusieurs sorties de terrain), ii) la comparaison des résultats de la classification sur les logiciels de télédétection et le logiciel de SIG.

Concernant l'élaboration de la carte de degré de risque de la dégradation de la forêt de la zone d'étude, elle consiste en l'identification, l'évaluation et l'intégration des facteurs physiques de base, tels que la topographie (pentes et exposition des versants à partir des cartes topographiques de Beni Mellal et Imilchil 1/100'000), la lithologie (carte de Beni Mellal et Imilchil 1/100'000), et le couvert végétal (images LandSat 1972-1999-2020) et travail de terrain, afin de fournir des informations sur l'état potentiel de la dégradation des versants ainsi les facteurs responsables à la dégradation de la forêt. 


\section{RESULTATS}

\subsection{Classification du couvert végétal de la zone d'étude}

Après avoir identifié les classes retenues, nous avons pris plusieurs échantillons en se basant sur les signatures spectrales du couvert végétal et des surfaces dépourvues de végétation. En effet, le traitement des images a montré une variation très importante de l'extension spatiale et de la superficie du couvert végétal entre les trois dates prédéfinies 1972, 1999 et 2020 (carte 2).

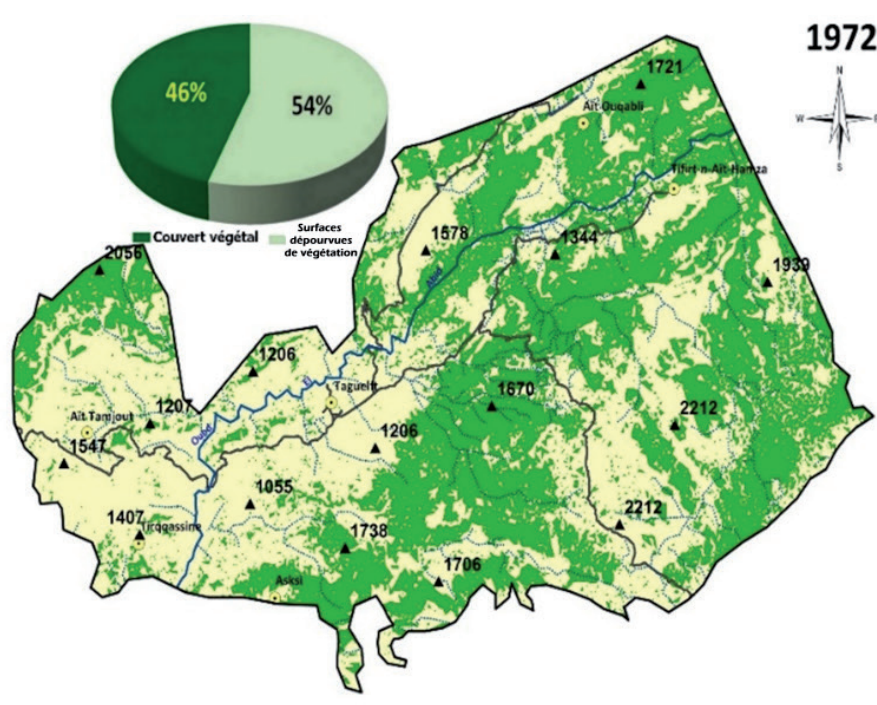

\section{Légende}

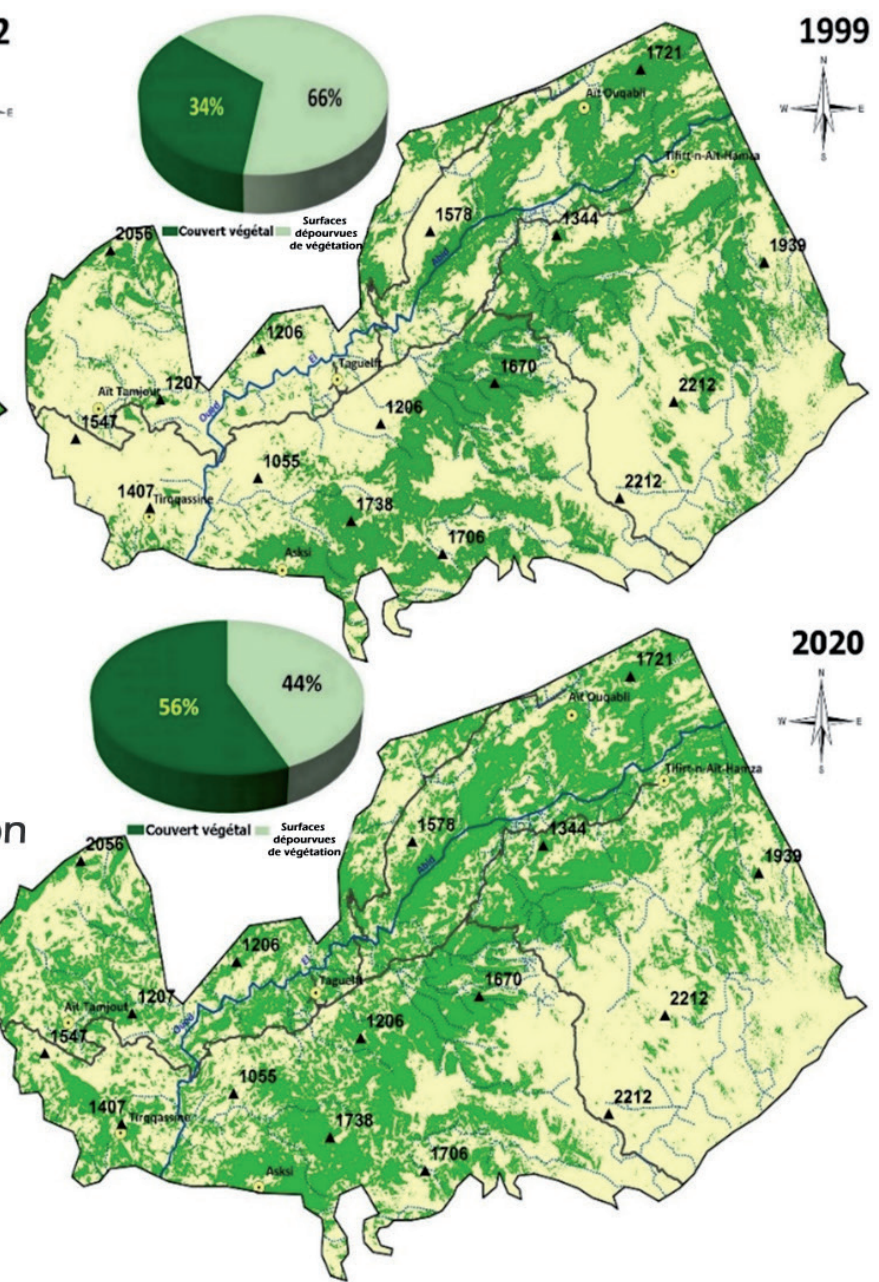

Carte 2. Dynamique du couvert végétal de l’image Landsat 1972, 1999 et 2020. Source: Élaboration des auteurs.

A la lumière des cartes de la classification, nous observons une dynamique spatio-temporelle très remarquable. Cette dynamique est résumée dans un grand recul du couvert végétal entre les deux années 1972 et 1999. Les zones qui ont connu une importante régression de la superficie du couvert forestier sont la partie Sud-Est et la partie Nord-Ouest de la zone de l'étude. En revanche, le couvert végétal de la forêt des Ait Daoud ou Ali a connu une progression entre les deux années 1999 et 2020, notamment au long de la direction et l'alignement Sud-Ouest Nord Est (spécialement au centre de la zone d'étude et sur les alentours des douars Asksi et Ait Tamjout d'une part, et sur les centres des communes de Taguelft, des Ait Ouqabli et de Tifirt n'Aït Hamza d'autre part).

Pour bien concrétiser et évaluer la dynamique du couvert végétal entre les trois dates retenues, nous avons représenté des résultats quantitatifs des variations du couvert végétal et des surfaces dépourvues de végétation tirés de la classification supervisée dans le tableau 2. On remarque qu'en 1972, superficie du couvert végétal à l'échelle de la zone de la forêt était de l'ordre de 14195.6 ha, et il se concentre essentiellement 
dans la partie centre et la partie Est de la zone de l'étude. En revanche, la partie Ouest, qui conforme le synclinal de Tagleft et la vallée de l'Oued El Abid, a connu une dominance des surfaces dépourvues de végétation dont la superficie est de 16664,4 ha. Quant à la classification effectuée pour l'année 1999, le couvert végétal montre un recul très important de point de vue surfacique par rapport à l'année 1972. Les zones ayant connues cette importante régression sont la partie centre et la partie Sud-Ouest de la zone de la forêt des Ait Daoud ou Ali. En effet, la superficie de la forêt a été réduite avec 12\% de la surface totale par rapport à l'année 1972. Par ailleurs, les surfaces dépourvues de végétation ont enregistré une progression très remarquable, dont ils atteignent 3703.2 ha.

Tableau 2. Changement du couvert végétal en 1972, 1999 et 2020.

\begin{tabular}{|c|c|c|c|c|}
\hline \multirow{2}{*}{ Années } & \multicolumn{2}{|c|}{ Couvert végétal } & \multicolumn{2}{|c|}{ Surfaces dépourvues de végétation } \\
\hline & $h a$ & $\%$ & ha & $\%$ \\
\hline 1972 & 14195,6 & 46 & 16664,4 & 54 \\
\hline 1999 & 10492,4 & 34 & 20367,6 & 66 \\
\hline 2020 & 17281,6 & 56 & 13578,4 & 44 \\
\hline Variation entre 1972-1999 & $-3703,2$ & -12 & 3703,2 & 12 \\
\hline Variation entre 1999-2020 & 6789,2 & 22 & $-6789,2$ & -22 \\
\hline Variation entre $1972-2020$ & 3086 & 10 & -3086 & -10 \\
\hline
\end{tabular}

Source: Élaboration des auteurs.

En effet, pendant les années 80, le Maroc a connu le déficit pluviométrique le plus important avec des périodes de sécheresse pénible (Hili et al., 2020; Bissour et al., 2018; Hanchan, 2014; Wassenburg et al., 2013; Benassi, 2008; Stour \& Agoumi, 2008; Karrouk, 2003), ces conditions ont influencé directement la répartition spatiale des précipitations, et par conséquent la spatialisation et l'évolution du couvert végétal. Pour cela, pendant ces années, les ressources naturelles en général, et le couvert végétal en particulier ont enregistré une surexploitation très abusive.

Quant à la classification effectuée pour l'image Landsat 2020, l'analyse de la carte obtenue de la dynamique du couvert végétal nous permet de souligner une importante progression de la densité de la forêt des Ait Daoud ou Ali. Les zones concernées par cette progression sont les alentours du centre de la commune de Tagleft, la partie Ouest près du douar des Ait Tamjout. Cette progression est de l'ordre de 10\% par rapport à l'année 1999. En revanche, nous remarquons sur la partie Sud-Est de la zone d'étude un remarquable recul du couvert végétal, malgré que les versants sont exposés vers le Nord et vers l'Ouest, où les sols sont riches en matière organique et en humidité. Normalement, la justification de cette progression est la déclaration de plusieurs lois, qui visent la protection de l'environnement.

Dans ce sens, le Royaume du Maroc a participé au "Sommet de la Terre" à Rio de Janeiro en 1992, delà et en raison de sa position géographique, le Maroc a proposé plusieurs actions de reboisement et autant de réglementations et de lois pour la conservation et la protection de la nature en général, et la richesse de sa diversité biologique en particulier à travers la création des aires protégées et des parcs nationaux.

Après la déclaration des lois (la loi 11-03 relative à la protection et à la mise en valeur de l'environnement, la loi 12-03 relative aux études d'impact sur l'environnement, et notamment la loi 22-07 relative aux aires protégées), nous avons remarqué une progression de la superficie du couvert végétal en faveur de la surface des surfaces dépourvues de végétation (carte 3). Cette augmentation est prouvée par l'intérêt donné à la protection et à la sauvegarde de la biodiversité à travers les différentes actions menées par les autorités chargées par la conservation des zones forestières (Direction des Eaux et Forêts). Malgré les efforts menés pour la protection, le reboisement et la progression enregistrée entre 1999 et 2020, le terrain de l'étude a enregistré encore un recul général entre 1972 et 2020 de l'ordre de 10\%. 


\subsection{La dynamique spatio-temporelle du couvert végétal}

Afin de montrer les zones de recul et de progression, nous avons élaboré les cartes de la dynamique du couvert végétal de zone de la forêt des Ait Daoud ou Ali, en croisant les trois cartes (1972-1999-2020) pour ressortir la carte de la dynamique spatio-temporelle de cette zone (carte 3). Dans ces cartes nous avons retenu quatre classes: surfaces dépourvues de végétation, la forêt, les zones de recul ou de dégradation et les zones de progression ou de croissance du couvert végétal et forestier.
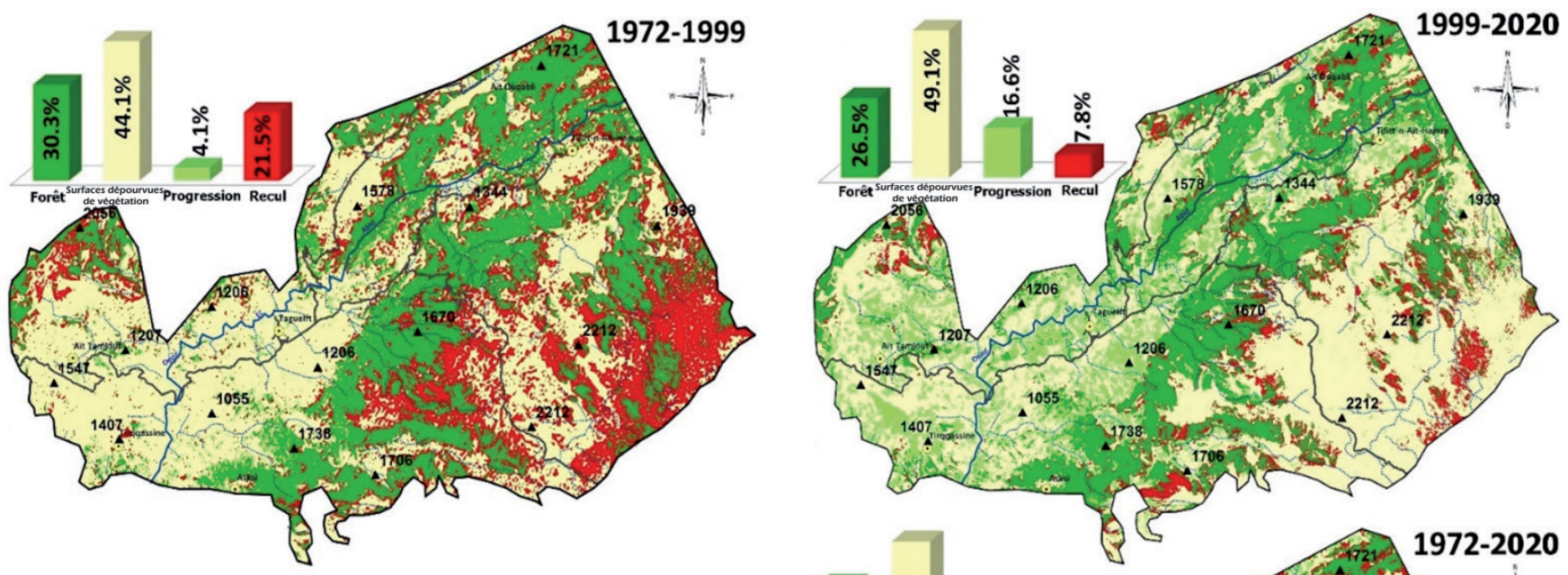

\section{Légende}

Zones de progression

Zones de recul

Surfaces dépourvues de végétation(Zones de stabilité)

Couvert végétal (Zones de stabilité)

$\Omega$ Limite de la forêt

$\sim$ Cours d'eau permanent

Cours d'eau temporaire

Réseau routier

- Ponits d'altitude

- Centres
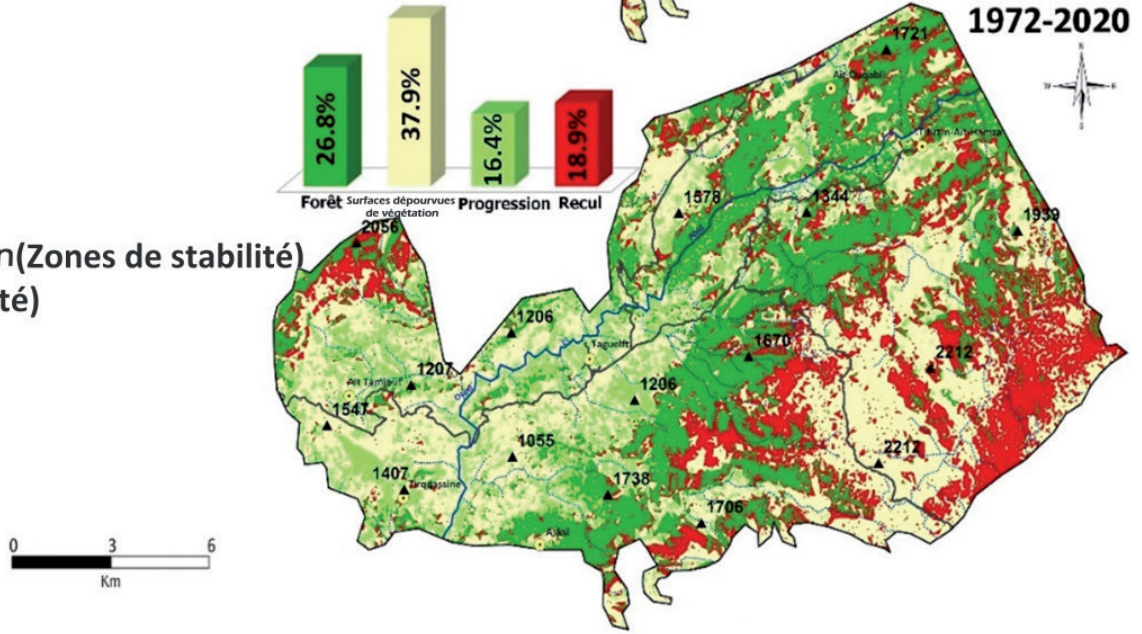

Carte 3. Zones de progression et de recul du couvert végétal de la forêt des Ait Daoud ou Ali. Source: Élaboration des auteurs.

Quant à la première carte, qui concerne l'évolution du couvert végétal entre 1972 et 1999, l'analyse des résultats de la carte nous permet de distinguer un recul important du couvert végétal notamment au centre, au Sud-Est et au Nord-Est de la zone de la forêt. En effet, ce recul marque une dégradation des zones couvertes autrefois par la forêt avec $21.5 \%$. Par contre, la progression de la forêt a touché des zones très limitées qui ne dépassent pas $4.1 \%$. Ainsi, les zones de stabilité (la forêt et les surfaces dépourvues de végétation) couvrent respectivement $30.3 \%$ et $44.1 \%$ (graphique de la carte 3, année 1972-1999).

Concernant l'analyse des résultats de la dynamique du couvert végétal des Ait Daoud ou Ali entre 1999 et 2020, durant cette période (période de promulgation des lois de la protection de la biodiversité et de l'environnement), les surfaces dépourvues de végétation ont régressé en faveur des surfaces de progression de la forêt avec 16.6\% notamment dans la partie centrale de forêt près de Tagleft et au Nord du douar Asksi, et à l'Ouest près des douars des Ait Tamjout et de Tirqqassine. Par ailleurs, les surfaces de recul et de dégradation ont enregistré une diminution au profit des surfaces dépourvues de végétation avec $7.8 \%$ notamment dans la partie Sud-Est de la zone. 
En effet, l'analyse des données de la classification de la période étudiée de 48 ans (entre 1972 et 2020), a montré que le couvert végétal de la zone d'étude a régressé dans l'ensemble en faveur des surfaces de reculs avec $18 \%$, par contre les surfaces dépourvues de végétation ont enregistré une régression en faveur des zones de progression avec $16 \%$.

Afin de vérifier et de valider les résultats de traitements des images (régression et/ou progression), nous avons i) effectué plusieurs sorties de terrain, dont nous avons enregistré une grande disposition spatiale entre la classification de l'évolution du couvert végétal, le degré de risque de la dégradation potentielle et la réalité de terrain. Ainsi, ii) ce constat a été vérifié par la comparaison des résultats de classification élaborée à l'aide de la Télédétection Spatiale (RS) et les Systèmes d'Information Géographique (GIS), dont nous avons noté une précision globale du traitement très satisfaisante (tableau 3).

Tableau 3. Comparaison des résultats de classification effectuée sur (RS) et (GIS).

\begin{tabular}{|c|c|c|c|c|c|c|}
\hline & \multicolumn{2}{|c|}{$\begin{array}{c}\text { Classification supervisée sur RS } \\
\text { "Region of interest (ROI)" }\end{array}$} & $\begin{array}{c}\text { Classification supervisée sur GIS } \\
\text { "Maximum Likelihood } \\
\text { Classification" }\end{array}$ & \multicolumn{2}{|c|}{ Différence } \\
\hline Années & $\begin{array}{c}\text { Couvert } \\
\text { végétal \% }\end{array}$ & $\begin{array}{c}\text { Surfaces } \\
\text { dépourvues de } \\
\text { végétation \% }\end{array}$ & $\begin{array}{c}\text { Couvert } \\
\text { végétal } \\
\%\end{array}$ & $\begin{array}{c}\text { Surfaces } \\
\text { dépourvues de } \\
\text { végétation \% }\end{array}$ & $\begin{array}{c}\text { Couvert végétal } \\
\%\end{array}$ & $\begin{array}{c}\text { Surfaces } \\
\text { dépourvues de } \\
\text { végétation \% }\end{array}$ \\
\hline 1972 & 45,82 & 54,17 & 46,1 & 53,9 & $\mathbf{0 , 2 8}$ (soit 86 ha) & $\mathbf{0 , 2 7}$ (soit 83 ha) \\
\hline 1999 & 34,13 & 65,85 & 33,82 & 66,17 & $\mathbf{0 , 3 1}$ (soit 96 ha) & $\mathbf{0 , 3 2}$ (soit 99 ha) \\
\hline 2020 & 56,04 & 43,94 & 55,78 & 44,21 & $\mathbf{0 , 2 6}$ (soit 80 ha) & $\mathbf{0 , 2 7}$ (soit 83 ha) \\
\hline
\end{tabular}

Source: Élaboration des auteurs.

La comparaison des résultats de la classification élaborée sous les deux logiciels, montre une faible différence de classification entre $0.26 \%$ et $0.32 \%$ entre les surfaces traitées et calculées. Pour ce fait, nous remarquons que le couvert végétal en 1972 s'étend sur une superficie de l'ordre de 45.82\% en utilisant l'outil "Region of Interest", et une superficie de $46.1 \%$ avec la méthode de "Maximum Likelihood Classification" sous l'outil SIG, avec une différence de $0.28 \%$. De même, les surfaces dépourvues de végétation enregistrent un écart de 83 ha soit $0.27 \%$. Pour la classification effectuée pour l'image de l'année 1999, la différence entre les zones a augmenté vers $0.31 \%$ pour le couvert végétal, et $0.32 \%$ pour les surfaces dépourvues de végétation. En revanche, pour l'année 2020, le couvert végétal représente un écart de $0.26 \%$ (soit 80 ha), et les surfaces dépourvues de végétation ont enregistré une variation de $0.27 \%$ soit 83 hectares.

En fait, l'existence de ces zones de différence est liée notamment à la présence de plusieurs pixels à la limite entre les zones du couvert végétal et les surfaces dépourvues de végétation. Dans ce sens, nous avons essayé de corriger au maximum les valeurs digitales de plusieurs pixels pour qu'ils reflètent la réalité du terrain et respectent les limites réelles de la forêt et des surfaces dépourvues de végétation.

\subsection{La dégradation potentiel du couvert végétal de la zone d'étude}

Pour vérifier la dynamique du couvert végétal de notre zone d'étude nous avons élaboré la carte de degré de risque de la dégradation potentiel du couvert végétal (carte 4). En effet, la superposition des facteurs naturels ayant une influence directe et indirecte sur l'évolution du couvert végétal nous a permet de hiérarchiser la carte de risque en potentiel faible à plus élevé (carte 4).

En effet, la carte 4, nous renseigne sur l'état de dégradation potentiel de la zone étudiée. D’après cette carte, nous constatons que la zone de la forêt est caractérisée par une dégradation potentielle très élevée. 


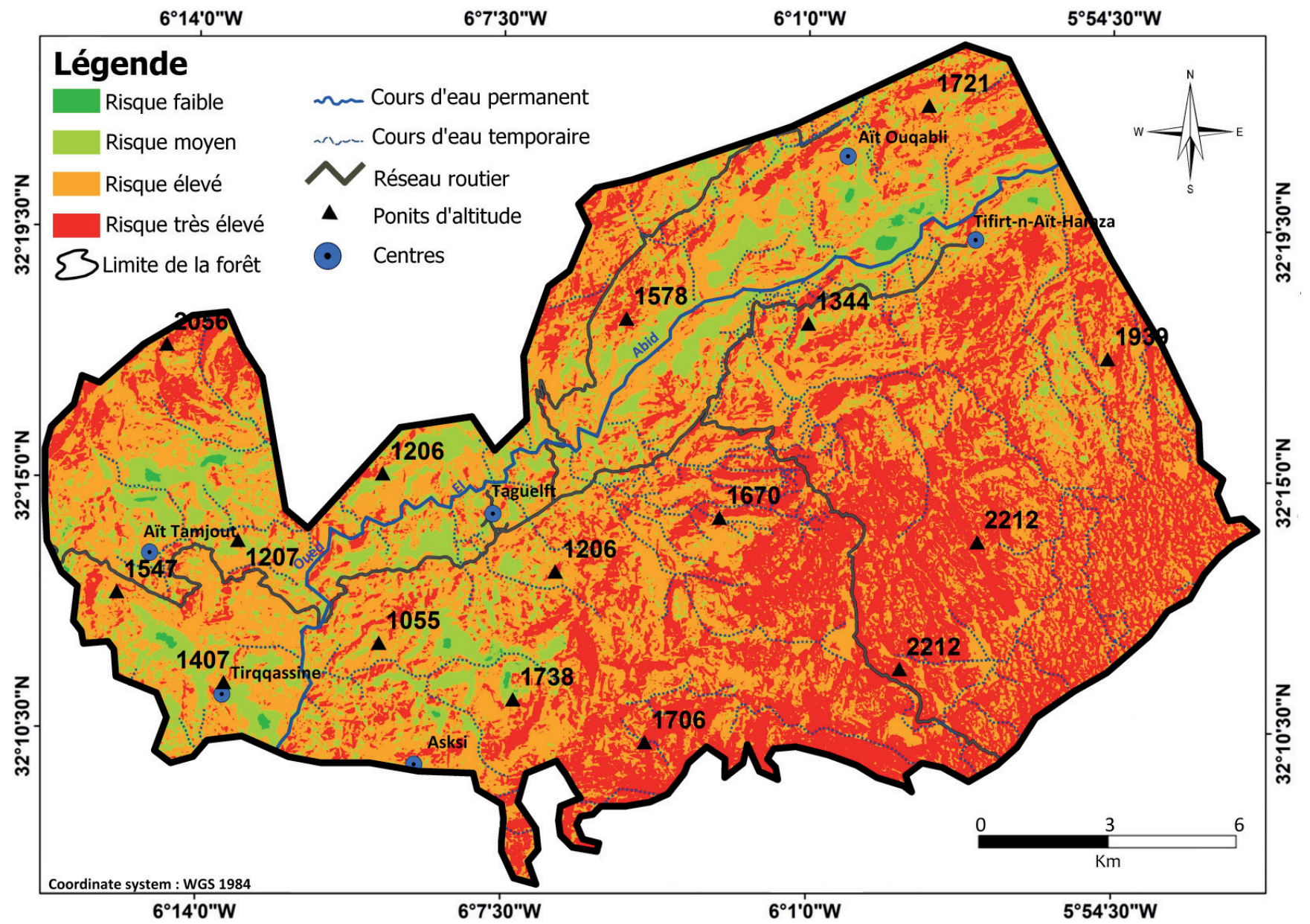

Carte 4. Degré de risque de la dégradation potentiel de la forêt des Ait Daoud ou Ali. Source: Élaboration des auteurs.

\subsection{Les pratiques anthropiques touchant l'intégrité de couvert végétal de la zone d'étude}

Pour monter le rôle des interventions humaines dans notre zone d'étude Les sorties de terrains et l'analyse de cette carte de degré de risque de la dégradation, montrent une grande coïncidence entre les zones à risque élevé et très élevé qui couvrent une superficie qui représente $72.3 \%$ avec les zones de recul, notamment à l'Est et au Sud-Est de la zone d'étude (Sud de Tifirt-n-Aït Hamza, Est d'Asksi, et Sud-Est de Taguelft). En revanche, les zones à risque faible (représentent $7.2 \%$ de la surface de la zone de la forêt) et moyen (couvrent 20.7\%) se coïncident avec les zones de stabilité et les zones de progression, cette coïncidence est bien visible à l'Ouest de Tifert n'Aït Hamza (Sud des Ait Ouqabli), au Nord d'Asksi, et Ait Tamjout, et au Sud-Ouest de Taguelft.

Généralement, les faibles quantités de précipitations, la topographie accidentée, la lithologie calcaire (Bissour, 2019) sont autant des facteurs qui sont à l'origine de la dynamique et l'évolution du couvert végétal. Outre les facteurs naturels, nous avons essayé d'évaluer l'effet des interventions anthropiques, l'analyse des données du questionnaire, nous a permis de dégager les actions et les interventions inappropriées les plus utilisées par l'Homme amenant à la dégradation du couvert végétal (Tra Bi,2013), l'utilisation du bois pour le charbonnage, pour la construction des maisons et pour le chauffage. De même, la population exerce une grande action négative sur la forêt par le surpâturage, et par l'effet de la déforestation et la conversion des terrains forestiers en des zones agricoles (Bouzekraoui et al., 2016; El Mazi et al., 2018). Ceci est bien claire dans les environs du douar d'Aglef (Plateau d'Aglef) et aux alentours du centre de Tifert n'Ait Hamza).

D’après la figure 2, nous remarquons que $32.1 \%$ de la population locale (questionnée) utilise les bois issus de la forêt des Ait Daoud ou Ali pour le chauffage et la cuisine. Ce pourcentage a pour origine le climat dur qui règne dans la zone de la forêt, notamment dans les parties Sud et Est, où les précipitations nivales sont 
très importantes pendant les périodes estivales et les minimas des températures sont souvent inférieurs à $0^{\circ} \mathrm{C}$ pendant les périodes humides, et peuvent durer plusieurs mois. De même, $29.7 \%$ de la population exerce un surpâturage au milieu de la forêt, ce problème pèse lourdement lorsque le troupeau comporte un nombre considérable de tète (plus de 200 têtes), et surtout s'il comporte des chèvres (figure 3), qui peuvent atteindre les parties supérieures des arbres et mangent notamment les nouveaux bourgeons des branches.

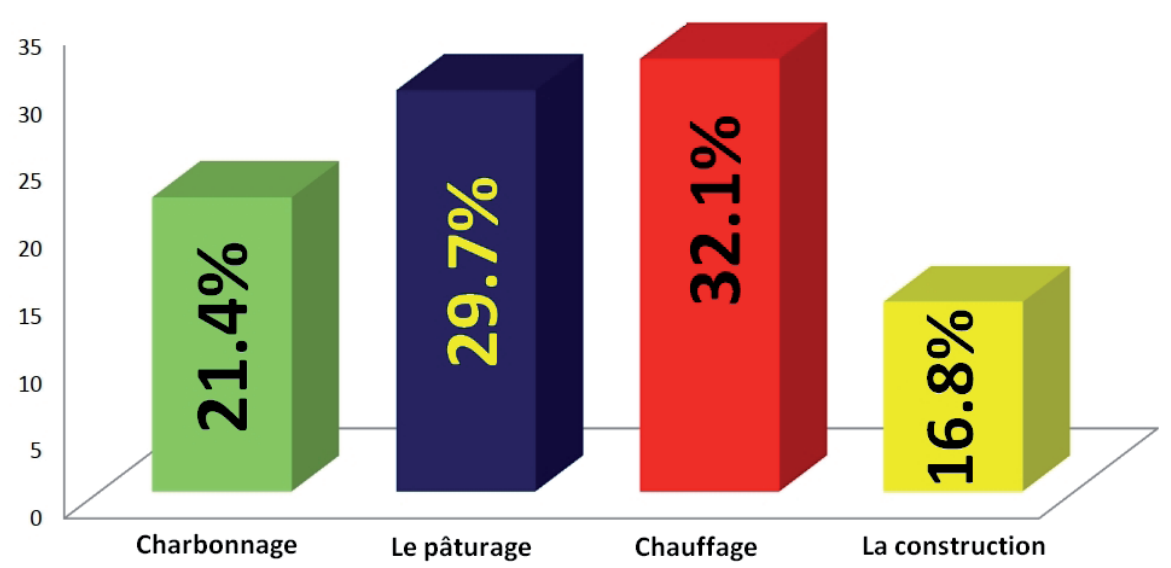

Figure 2. Actions anthropiques dégradant le couvert végétal de la zone d’étude. Source: Élaboration des auteurs.

Outre cela, $21.4 \%$ de la population pratiquent le charbonnage notamment avec le chêne vert, car il est examiné et considéré comme un bois très excellent pour cette opération. Selon les responsables des Eaux et Forêts rencontrés, plus de $210 \mathrm{Kg} / \mathrm{st}$ du bois sont exploités pour le charbonnage. Cette action est exercée par la population locale pour des fins commerciaux, dont le charbons produit est souvent destiné aux centres ruraux (Tagleft, Larbaa n'Ait Ouqabli, Tifert n'Ait Hamza, Ouaouizerht, Aghbala, El Ksiba) et villes comme Beni Mellal dans la plaine de Tadla. Malgré les efforts déployés par les autorités chargées de la protection et la garde de la forêt pour lutter contre le charbonnage illégal et non autorisé, le charbonnage continue à exercer son effet négatif sur la forêt des Ait Daoud ou Ali, en attendant que la population locale dispose d'autre ressources économiques. Enfin, 16.8\% coupe le bois de la forêt pour la construction des maisons (figure 4). Cette portion est due généralement à la nature et type des constructions, dont la population n'utilise que les sols (argiles ou marnes), des pierres et du bois pour la construction de leurs abris.

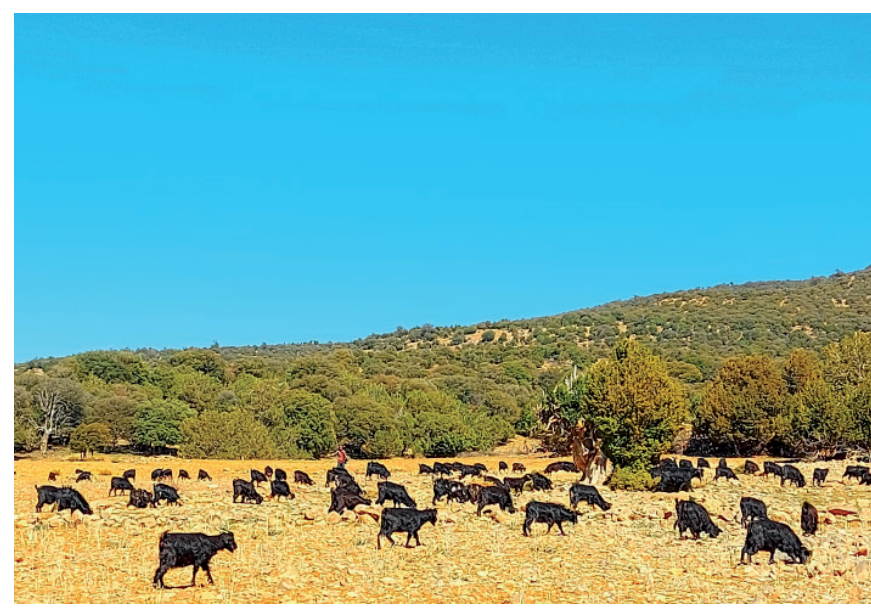

Figure 3. Le surpâturage des chèvres dans la forêt. Source : Hili, 2020.



Figure 4. La construction des maisons. Source: Hili, 2020. 


\section{DISCUSSION}

En général, la répartition et la dynamique du couvert végétal au Maroc (Hili \& Abdelaziz, 2021; Bissour et al., 2018; Sbai et al., 2018; El Mazi et al., 2018; Bouzekraoui et al., 2016; Daoui \& Fatemi, 2014; Tra Bi, 2013; Hammi, 2007; Labhar, 1998; Benabid, 1985; Lecompte, 1986; Michel \& Ruelan, 1967) comme les autres pays du monde (Gansaonré et al.; 2020, Raogo, 2018; Diop et al., 2018; Soro et al., 2014; Bouiadjra et al., 2011; Dawson et al., 2011; Brou, 2010; Mahamane et al., 2007; Demarteau et al., 2007; Ouloukoi et al., 2006; Jeffrey et al., 2006; Cabid et al., 2005; Hountondji et al., 2004; Carmen Salazar Conde et al., 2004; Nguessan, et al., 2003; Demaze, 2003; Dupouey et al., 1999; Thimonier, 1994) sont liées aux changements environnementaux et/ou interventions anthropiques, dans notre terrain d'étude, deux types de causes possibles peuvent expliquer ces changements observés du couvert végétal entre les trois dates étudiées.

Pour la période entre 1972 et 1999, les changements observés du couvert végétal sont liés aux facteurs naturels notamment les conditions climatiques défavorables qu'a connu le Maroc (les périodes de la sécheresse) en général et de la zone d'étude en particulier. Cette période a été marqué par un stress climatique et hydrique très remarquable qui a touché la dynamique spatio-temporelle du couvert végétal dans cette zone. En revanche, entre 1999 et 2020, le Maroc en général a enregistré une augmentation de la démographie, ce qui a changé les besoins et les pratiques de l'Homme, pour cela malgré la promulgation des lois de la protection de la biodiversité et de l'environnement, les interventions de l'état (reboisement) et les conditions climatiques plus ou moins favorables les actions anthropiques ont laissé un impact majeur sur la dynamique et la répartition du couvert végétal (Hili \& Abdelaziz, 2021; Bouzekraoui et al., 2016; Tra Bi, 2013; Nguessan et al., 2003). Les principaux résultats ont indiqué un vrai recul du couvert végétal issue à une dégradation de plus en plus importante du milieu liée notamment à la demande intense sur le bois et aussi aux pratiques anthropiques (le pâturage, charbonnage, agropastorale, ...).

En effet, l'étude de la dynamique spatio-temporelle du couvert végétal de la forêt des Ait Douad ou Ali a montré que les facteurs naturels tels que le climat (précipitations - surtout nivales-, température, etc.), la topographie (notamment les altitudes, l'exposition des versants et les pentes), la lithologie sont autant de facteurs qui ont influencé et influencent encore la répartition spatiale et la dynamique du couvert végétal de la forêt des Aït Douad ou Ali.

Ainsi, l'évolution des pratiques anthropiques (le pâturage, charbonnage, ...) sur le territoire d'étude est considérée comme un facteur très important pour expliquer la dynamique du couvert végétal de la forêt des Ait Daoud ou Ali observée pendant ces dernières 48 ans. Dans ce sens, nous avons distingué quatre usages principaux qui peuvent être responsables à la dégradation du couvert végétal dans notre zone d'étude, ces quatre actions sont variables dans le temps et dans l'espace selon les besoins de l'Homme.

Il faut dire que les études que nous avons mené sur la dynamique spatio-temporelle du couvert végétal sur quelques zones de la région de Beni Mellal-Khènifra, notamment sur la commune d'Aghbala (en cous de publication) et sur la forêt des Ait Bouzid (fera l'objet d'un autre article), penchent vers une tendance d'une forte dégradation et d'une forte régression du couvert végétal dans les trois zones (forêt des Ait Daoud ou Ali, commune d'Aghbala, la forêt des Ait Bouzid). Cette tendance de dégradation est liée essentiellement dans ces trois zones aux actions anthropiques inappropriées, très intensives et inconscientes.

\section{CONCLUSION}

La classification multi-chronique $(1972,1999$ et 2020) menée dans ce travail a montré que la zone de la forêt des Ait Daoud ou Ali a connu une évolution très remarquable durant les 48 ans dernières.

Durant cette période, notamment entre 1972 et 1999, nous avons enregistré un fort recul de la superficie du couvert végétal en faveur des surfaces dépourvues de végétation avec $12 \%$ au Sud-Est de la zone d'étude. Cette diminution, est impliqué dans un contexte climatique particulier, où le Maroc a connu des longues périodes de sécheresse (les années 1980). Après cette période, notamment entre 1999 et 2020, le Maroc s'est engagé à proposer et adopter plusieurs réglementations et lois, pour la protection de la nature en général, 
et de la richesse de sa diversité biologique en particulier. Certes, cette politique a donné ces fruits quelques années plus tard (même si qu'elle reste très modeste à l'échelle du Haut Atlas Central et à la région de Beni Mellal-Khénifra en particulier). Sur la zone de la forêt qui fait objet de cette étude, nous pouvons remarquer une progression du couvert végétal (en général) avec $22 \%$. Malgré cette progression, l'aire de l'étude enregistre encore une perte de $10 \%$ de son couvert végétal.

Les résultats de classification obtenus sont satisfaisants, sa validation a été vérifiée par l'observations directes de la spatialisation des zones lors des sorties de terrains, et aussi par la comparaison des résultats obtenus de la classification de l'outil "Region of Interest" utilisé sous les outils de la télédétection spatiale, avec celle de la classification "Maximum Likelihood Classification" effectué à l'aide des Systèmes d'Informations Géographiques. L'étude de ces deux méthodes a montré une précision élevée du traitement globale, avec une faible différence entre les zones qui varie entre $-0.26 \%$ et $0.32 \%$.

En général, il faut dire que l'évolution spatio-temporelle du couvert végétal est influencée et liée essentiellement à la conjonction de plusieurs facteurs naturels (le climat, les altitudes, l'exposition des versants et les pentes, la lithologie) et anthropiques.

Cette étude a mis en évidence l'impact des actions anthropiques notamment l'utilisation du bois pour le charbonnage, pour la construction des maisons, pour le chauffage (utilisation socio-économique de la ressource forestière) ainsi que l'impact de surpâturage et l'activité agricole dans la zone forestière (agroforestier), vu que l'activité principale de la population locale se résume dans l'agriculture et l'élevage). L'utilisation des techniques géospatiales nous a permis d'avoir des informations très précises relatives à l'évolution spatio-temporelle de recul et aussi de la progression du couvert végétal dans la forêt des Ait Daoud ou Ali.

Pour cela, on peut considérer que la méthode utilisée dans cette étude, qui associer à la fois l'analyse, l'interprétation visuelle et les traitements numériques, est satisfaisante et opérationnelle, elle peut donc être appliquée sur l'ensemble des études dédiées au suivi de la dynamique spatio-temporelle du couvert végétal.

\section{Signalement responsable et conflit d'intérêts}

Tous les auteurs s'engagent à divulguer tout conflit d'intérêts existant ou potentiel en relation avec la publication de notre article. De même, les tâches effectuées par les auteurs sont i) pour Aïman HILI, Rachid BISSOUR et Yassine El Jouhary, ils ont fait la collecte des données (cartes, images satellites, précipitations, ...), l'analyse des données, les sorties de terrain, l'élaboration des cartes et des bases de données, et la vérification des résultats sur le terrain; ii) pour les auteurs Aïman HILI, Fardi JAA et Hanane REDDAD ils ont fait l'orientation et l'encadrement du travail, l'analyse des données, la correction du manuscrit et la validation des résultats.

\section{RÉFÉRENCES}

Bahzad, H. (1982). Recherches hydrologiques et hydrochimiques sur quelques karsts méditerranéens: Liban, Syrie et Maroc. Livre deuxième. (Thèse de doctorat d'état), Université d'Aix-Marseille II, Institut de Géographie.

Benabid, A. (1987). Les grands écosystèmes terrestres et leur préservation. G.E.M. Rabal, 4, 77-190.

Benassi, M. (2008). Drought and climate change in Morocco. Analysis of precipitation field and water supply. In A. López-Francos (ed.). Drought management: scientific and technological innovations (pp.83-86). CIHEAM, 2008. Options Méditerranéennes: Série $A, \mathrm{n}^{\circ} 80$.

Bissour, R. (2019). Ressources en eau de l'oued El Abid amont barrage Bin El Ouidane et agriculture irriguée: Caractérisation, quantification et valorisation. Cas du périmètre irrigué de Beni Moussa (Région Beni Mellal - Khénifra). (Thèse de doctorat). Université Sultan Moulay Slimane, Beni Mellal, Maroc.

Bissour, R., \& El Khalki, Y. (2018). Fluctuations pluviométriques et leurs impacts sur le régime hydrologique du bassin versant de l'oued El Abid en amont du barrage de Bin El Ouidane (Haut Atlas Central). Acte du colloque Climat, Eau et Société (CES'2015); De la gestion des ressources en eau à la gestion des systèmes aquatiques, 1, 11-26.

Bissour, R., El Khalki, Y., \& Hili A. (2018). Apport de la télédétection spatiale à l'étude diachronique de la dynamique de l'occupation du sol dans le bassin versant de l'Oued El Abid, (Haut Atlas central, Maroc). Actes du colloque International des utilisateurs du SIG, Oujda. 
Bouiadjra, S., El Zerey, W., \& Benabdeli, K. (2011). Étude diachronique des changements du couvert végétal dans un écosystème montagneux par télédétection spatiale: cas des monts du Tessala (Algérie occidentale). Varia, 5, 211225. https://doi.org/10.4000/physio-geo.2048

Bouzekraoui, H., El Khalki, Y., Mouaddine, A., Lhissou, R., El Youssi, M., \& Barakat, A. (2016). Characterization and dynamics of agroforestry landscape using geospatial techniques and field survey: a case study in central High-Atlas (Morocco). Agroforestry Systems. 90 (6), 965-978. https://doi.org/10.1007/s10457-015-9877-8

Brou, T. (2010). Variabilité climatique, déforestation et dynamique agrodémographique en Côte d'Ivoire. Sécheresse, 21(1e), 1-6. https://doi.org/10.1684/sec.2010.0266

Cabid, M., Zak, M., R., Cingolani, A., Cáceres, D. \& Díaz, S., (2005). Cambios en la cobertura de la vegetación del centro de la Argentina. ¿Factores directos o causas subyacentes? https://www.researchgate.net/profile/Daniel-M-Caceres/publication/323548160.

Carmen Salazar Conde, E., Zavala Cruz, J., Castillo Acosta, O., et Cámara Artigas, R. (2004). Evaluación espacial y temporal de la vegetación de la Sierra Madrigal, Tabasco, México (1973-2003). Investigaciones Geográficas, Boletín del Instituto de Geografía, 54. 7-23.

Combe, M. (1977). Haut Atlas calcaire. Ressources en eau du Maroc, domaines atlasiques et sud-atlasiques. Tome III. Notes et mémoires du Service Géologique. Maroc, n²31, 116-139.

Couvreur, G., (1988). Essai sur l'évolution morphologique du Haut Atlas central calcaire (Maroc). Notes et Mémoires du Service géologique, $\mathrm{n}^{\circ} 318$.

Daoui, K., \& Fatemi, Zea. (2014). Agroforestry systems in Morocco: the case of olive tree and annual crops association in Saïs region. In M. Behnassi et al (eds.) Science, policy and politics of modern agricultural system (281-290). Springer. https://doi.org/10.1007/978-94-007-7957-0_19

Dawson, IK., Vinceti, B., Weber, JC., Neufeldt, H., Russell, J., Lengkeek, AG., Kalinganire, A., Kindt, R., Lilles, JPB. \& Roshetko, J. (2011). Climate change and tree genetic resource management: maintaining and enhancing the productivity and value of smallholder tropical agroforestry landscapes: a review. Agroforestry Systems, 81, 67-78. https://doi. org/10.1007/s10457-010-9302-2

Demarteau, M., Francois., L. Cheddadi, R. \& Roche., E. (2007). Réponses de Cedrus atlantica aux changements climatiques passés et futurs. Geo-Eco-Trop, 31, 105-146.

Demaze, M.T. (2003). Caractérisation et suivi de la déforestation en milieu tropical par télédétection. Application aux défrichements agricoles en Guyane française et au Brésil. Télédétection, 3(5 ), 477-478.

Diop, A., Hyacinthe. S., Cheikh, D., Elysée, N., Honoré, D. \& Bienvenu, S. (2018). Dynamique d'occupation du sol des zones humides urbanisées de Dakar (Sénégal) de 1942 à 2014. VertigO - la revue électronique en sciences de l'environnement, 18. https://doi.org/10.4000/vertigo.20120

Direction Régionale des Eaux Et Forêts, Beni Mellal. (2010). Etude d'aménagement de la forêt des Ait Daoud Ouami, Rapport de synthèse.

Dupouey, J.-L., Thimonier A., Lebourgeois F., Becker M., F.Picard J. \& Timbal J. (1999). Changements de la végétation dans les forêts du nord-est de la France entre 1970 et 1990. Rev.For.Fr.LI, 2, 219-230. https://doi.org/10.4267/2042/5432

El Mazi, M., Saber, E. \& Houari, A. (2018). Evolution spatio-temporelle des écosystèmes forestiers dans les massifs numidiens de la chaîne rifaine (Maroc): cas de Jbel Outka. Geo-Eco-Trop, 42 (1), 133-146.

Gansaonré, R.N., Benewindé, J.-B., Zoungrana \& Isidore Yanogo, P. (2020). Dynamique du couvert végétal à la périphérie du Parc W du Burkina Faso. Belgeo. https://doi.org/10.4000/belgeo.40786

Hammi, S. (2007). Evolution des recouvrements forestiers et d'occupation des sols entre 1964 et 2002 dans la haute vallée des Ait Bougumez (Haut Atlas central, Maroc). Sécheresse, 18, 1-7.

Hanchan, M. (2014). Impact du changement climatique sur les précipitations au Maroc: de la modélisation prospective à l'observation des séries temporelles actuelles. Cas de Taza et Berkine. Actes du colloque, Taza et son arrière-pays: ressources territoriales et perspectives du développement durables (95-118).

Hili, A., Bissour R. \& El Khalki, Y. (2020). The influence of climatic variations on geotourism in the National Parc of Tazekka (middle eastern north Atlas), which adaptation? Actes du colloque international Climat, Eau et Société (CES'2019) (71-76).

Hili, A. \& Abdelaziz, R. (2021). Apport du SIG et de la télédétection à l'étude de la dynamique du couvert végétal dans la commune d'Aghbala, province de Beni Mellal. Revue GéoDev, (accepté, en cours de publication) - Texte en arabe. 
Hili, A. \& El Khalki, Y. (2017). Les paysages karstiques du Parc National de Tazekka (Moyen Atlas nord oriental, province de Taza): inventaire, évaluation et valorisation pour un développement territorial durable et intégré. Karstologia,70, 43-52.

Hountondji, Y.C., Ozer, P. \& Nicolas, J. (2004). Mise en évidence des zones touchées par la désertification par télédétection à basse résolution au Niger. Cybergeo: European Journal of Geography, Environnement, Nature, Paysage, 291,1-18. https://doi.org/10.4000/cybergeo.2761

Jeffrey, A., Mcneel, Y. \& Schroth, G. (2006). Agroforestry and biodiversity conservation-traditional practices, present dynamics, and lessons for the future. Biodiversity and Conservation, 15, 549-554. https://doi.org/10.1007/s10531005-2087-3

Karrouk, M.S. (2003). Dynamiques des climats au Maroc. (Thèse de doctorat d'état). Université Hassan II, FLSH Ben M'sick Casablanca.

Labhar, M. (1998). Les milieux forestiers et pré-forestiers du Moyen Atlas central nord occidental: Approche Géographique, phytoécologique et dynamique. (Thèse de Doctorat d'Etat). Université Libre de Bruxelles.

Lecompte, M. (1986). Biogéographie de la montagne marocaine: le Moyen Atlas central. Mémoires et documents de Géographie. Edit. CNRS.

Lecompte, M., \& Alexandre, F. (1996). Discontinu et continu de la végétation et du milieu: De la théorie des étagements en altitude à la phytoclimatologie dynamique. L'Espace géographique, 25(3), 261-272. https://doi.org/10.3406/spgeo.1996.995

Loi $n^{\circ}$ 11-03. (2003). La loi relative à la protection et à la mise en valeur de l'environnement. Bulletin Officiel.

Loi $n^{\circ}$ 12-03. (2003). La loi relative aux études d'impact sur l'environnement. Bulletin Officiel.

Loi $n^{\circ} 22-07$. (2010). La loi relative aux aires protégées. Bulletin Officiel.

Mahamane, A., Mahamane, S., Yacoubou, B., Issaka, A., Ichaou, A., \& Saley, K. (2007). Analyse diachronique de l'occupation des terres et caractéristiques de la végétation dans la commune de Gabi (région de Maradi, Niger). Sécheresse, 18(4), 296-304.

Michel, C. \& Ruelan, A. (1967). L'agriculture et les forêts au Maroc. Les cahiers de la recherche agronomique, $\mathrm{n}^{\circ} 24, \mathrm{R}^{2} \mathrm{bat}$, Maroc, Chapitre IV (103-140).

Monbaron, M. (1982). Un relief anti-bathonien enfoui sur la ride du Jbel La'bbadine (Haut Atlas central, Maroc): conséquences pour la chronologie de l'orogenèse atlasique. Bull. Ver. Schweiz. Petroleum-Geol. u. -Ing., 48(114), 9-25.

Nguessan, É., Bellan, M.F. \& Blasco, F. (2003). Suivi par télédétection spatiale d'une forêt tropicale humide protégée soumise à des pressions anthropique. Télédétection, 3 (5), 443 -456.

Ouloukoi, J., Mama, V.J. \& Bernadin, A.F. (2006). Modélisation de la dynamique de l'occupation des terres dans le département des collines au Benin. Télédétection. 2006, 6(4), 305-323.

Perritaz, L. (1995). Contribution à l'étude géomorphologique et hydrogéologique d'un Karst perché en domaine méditerranéen: le plateau des Aït Abdi: Haut Atlas central calcaire, Maroc. (Thèse doctorat). Université Fribourg. https://doi. org/10.3406/karst.1996.2372

Qi, J., Chehbouni, A., Huete, A.R., Kerr, Y.H., et Sorooshian, S. (1994). A Modified Soil Adjusted Vegetation Index. Remote Sensing of Environment, 48, 119-126. https://doi.org/10.1016/0034-4257(94)90134-1

Raogo, N. G. (2018). Dynamique du couvert végétal et implications socio-environnementales à la périphérie du parc W/Burkina Faso. Vertigo - la revue électronique en sciences de l'environnement, 18(1) https://doi.org/10.4000/vertigo.20249.

Rondeaux, G., Steven, M. \& Baret, F. (1996). Optimization of Soil-Adjusted Vegetation Indices. Remote Sens. Environ, 55, 95-107. https://doi.org/10.1016/0034-4257(95)00186-7

Sbai, A., Lasgaa, H. \& Sabri, M. (2018). Contribution de la télédétection dans l'étude et l'évolution spatiotemporelle du couvert végétal: cas du couloir de Taourirt - El Aioun et ses bordures montagneuses (Maroc oriental). Actes du coIloque International des utilisateurs du SIG, Oujda (245-250).

Soro, G., Ahoussi, E.K., Kouadio, E.K., Soro, T.D., Oulare, S., Saley, M.B., Soro, N. \& Biemi, J. (2014). Apport de la télédétection à la cartographie de l'évolution spatio-temporelle de la dynamique de l'occupation du sol dans la région des Lacs (Centre de la Côte d'Ivoire). Afrique Science, 10(3), 146-160.

Stour, L. \& Agoumi, A. (2008). Sécheresse climatique au Maroc durant les dernières décennies. Hydroécol. Appl. Tome 16 (pp. 215-232). https://doi.org/10.1051/hydro/2009003 
Thimonier, A. (1994). Changements de la végétation et des sols en forêt tempérée européenne au cours de la période $1970-$ 1990: rôle possible des apports atmosphériques. Sciences de l'environnement. Université Paris XI Orsay. 〈tel-02923657〉.

Tra Bi, Z.A., (2013). Etude de l'impact des activités anthropiques et de la variabilité climatique sur la végétation et les usages des sols, par utilisation de la télédétection et des statistiques agricoles, sur le bassin versant du Bouregreg (Maroc). (Thèse de doctorat). Université Félix Houphouët-Boigny (Abidjan, Côte d'Ivoire).

Wassenburg, J.A., Immenhauser, A., Richter, D.K., Niedermayr, A., Riechelmann, S., Fietzke, J., Scholz, D., Jochum, K.P., Fohlmeister, J., Schröder-Ritzrau, A., Sabaoui, A., Riechelmann, D.F.C., Schneider, L. \& Esper, J. (2013). Moroccan speleothem and tree ring records suggest a variable positive state of the North Atlantic Oscillation during the Medieval Warm Period. Earth and Planetary Science Letters. http://dx.doi.org/10.1016/j.epsl.2013.05.048 